\title{
Simultaneous temperature and strain measurement with enhanced resolution up to 20 times by using a compact hybrid optical waveguide Bragg grating
}

\author{
Kaiming Zhou, Xianfeng Chen, David Webb, Lin Zhang, Ian Bennion \\ Photonic Research Group, Aston University \\ k.zhou@aston.ac.uk
}

\begin{abstract}
A hybrid waveguide Bragg grating in optical fiber was fabricated and characterized, showing thermal responsivity of $211 \mathrm{pm} /{ }^{\circ} \mathrm{C}$. Proposed being used in fiber sensor, it demonstrates enhanced resolution by $20 \times$ and $2 \times$ for temperature and strain, respectively. (C2008 Optical Society of America

OCIS codes: (060.2370) Fiber optics sensors; (060.3735)_Fiber Bragg gratings
\end{abstract}

\section{Introduction}

Fiber Bragg gratings (FBGs) have been used extensively in optical fiber sensors, particularly for strain related applications. However, spectral response of FBGs to strain is usually mixed with that caused by temperature, and this cross-sensitivity problem still remains a technical issue requiring for practical solutions. The difficulty lies on that most FBGs present similar optical properties from device to device. Measures have to be taken to bring the diversity and there have been reports of using FBGs with different characteristics, by employing the different ratios of the strain response to the temperature response of each FBG[1-3], however, the ratio differences have been generally low. Long period gratings (LPGs) are another suitable solution for such a purpose since they are very sensitive to temperature but not to strain. Though LPGs have been reported for temperature discrimination [4], they have their own limitations and drawbacks. LPGs are usually large sized (several centermeters long) compared to FBGs and thus unsuitable for situations where miniature device is desirable. Spectra of LPGs are much broader than that of FBGs and give rise to technical challenge for interrogation, and are also very susceptible to bending, which generally accompanies with the measurand.

Recently, FBGs were also inscribed in polymer optical fibers and they present widely different optical characteristics to those in silica optical fibers [5], e.g. showing large thermal coefficient. It is thus very desirable to combine the FBGs in polymer and silica fibers for stain sensing with temperature discrimination capability by exploiting the large difference in their thermal coefficients. However, their physical properties are so different that it is even impossible to splice them together.

Femtosecond (fs) laser technology has shown great promise in making microstructures in variety materials, especially for those which are used to be hard to manipulate, such as glass, ceramic etc. Tightly focused fs laser pulses have been used to induce refractive index changes for writing optical waveguides and, with the aid of chemical etching, have been applied in micromachining. Hole-type features can be created on the surface of the optical fibre and we have developed a technique to make modification throughout the fiber using fs-inscription [6,7]. In this paper, we report on the creation of a novel in-fiber hybrid waveguide grating (HWG) structure by using the fs technique, which has a polymer core and a glass cladding. The usage of the HWG in a strain sensor with temperature discrimination is also suggested.

(a)

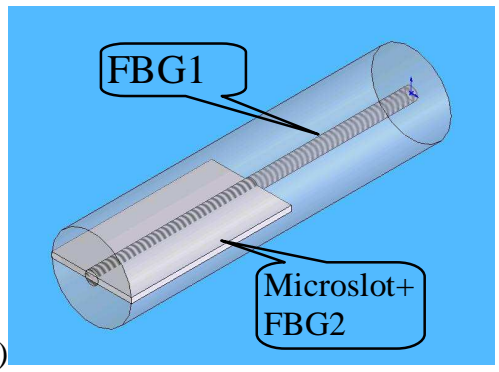

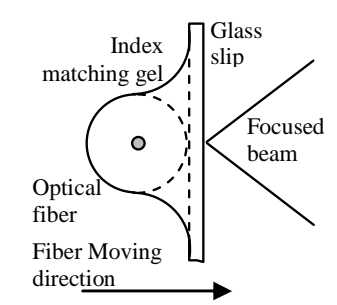

(b)

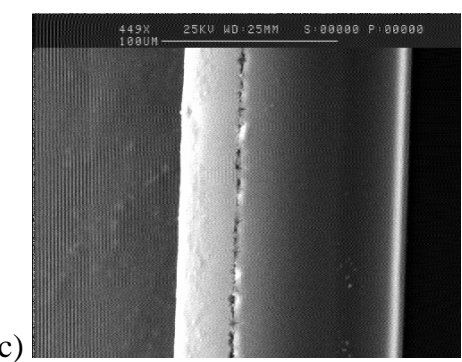

(c)

Fig. 1. (a) Sketch of the sensor concept which consists of a normal FBG for strain test and a micro slot based hybrid waveguide grating structure for temperature monitoring (b) Sketch of inscription of microstructures with tightly focused femtosecond laser in optical fiber which is index matched to a planar glass slip.(c) Scanning electron microscopy (SEM) of the micro-slot. 


\section{Description of the device and principal of operation}

Figure 1 shows the sketch of the hybrid optical waveguide and the operation principle of the sensor. The sensor consists of two neighbouring FBGs, FBG1 and FBG2. FBG1 is a normal FBG in glass optical fiber while the other is used to produce the hybrid waveguide grating (HWG), which is based on a slab waveguide structure with the glass cladding and the polymeric core. The slab waveguide is made by engraving a microslot through FBG2 with thickness smaller than the diameter of the fiber core and then filling it with polymer of higher refractive index. Hence, the HWG is more polymer-inclined and shows enhanced thermal sensitivity compared to FBG1.

When temperature variation and strain are applied, the Bragg wavelength shifts of these two grating are: $\Delta \lambda_{B, i}=K_{\varepsilon, i} \Delta \varepsilon+K_{T, i} \Delta T$ where $i=1,2$ is designated for HWG(FBG1) and FBG2 respectively. $K_{\varepsilon, i}$ and $K_{T, i}$ are the strain coefficient and thermal coefficient of these two gratings, respectively. With this relation, we can determine the temperature and strain applied to the fiber according to the gratings' spectral response by:

$$
\left(\begin{array}{c}
\Delta T \\
\Delta \varepsilon
\end{array}\right)=\frac{1}{M}\left(\begin{array}{cc}
K_{\varepsilon, 2} & -K_{\varepsilon, 1} \\
-K_{T, 2} & K_{T, 1}
\end{array}\right) \cdot\left(\begin{array}{l}
\Delta \lambda_{1} \\
\Delta \lambda_{2}
\end{array}\right)
$$

where $M=K_{\varepsilon, 2} K_{T, 1}-K_{\varepsilon, 1} K_{T, 2}$. With a certain resolution to $\Delta \lambda_{1}$ and $\Delta \lambda_{2}$, say 0.1 nm for most optical spectral analysers, the resolution for discernable variation of temperature and strain can be given by:

$$
\delta T=\frac{1}{M}\left(\left|K_{\varepsilon, 2}\right|+\left|K_{\varepsilon, 1}\right|\right) \cdot 0.1 \mathrm{~nm}, \delta \varepsilon=\frac{1}{M}\left(\left|K_{T, 2}\right|+\left|K_{T, 1}\right|\right) \cdot 0.1 \mathrm{~nm}
$$

Apparently the bigger of the absolute value of $M$ is, the better resolution will be achieved.

\section{Micromachining of the microslot through the optical fiber}

Figure 1(b) illustrates the optical geometry of the micromachining of the slot. A glass slip was adopted with index matching oil to eliminate the curvature of the fiber, yielding perfect modification in the whole region of the fiber[6]. With well alignment of the focal spot of the laser with respect to the fiber, a $125 \times 500 \mu \mathrm{m}$ rectangle patch was then scanned along the fiber axis and through the diameter of the fiber, generating a master pattern for the slot as shown in Fig. 1(a). For better interception of light from the untouched fiber section, the modification was made symmetrically across the core. The modification was also purposely made in the entire length of the FBG to maintain the uniformity of the grating when it was treated by fs laser and later chemical etching, avoiding unwanted interference on its spectrum.

Following the fs-laser inscription, the fiber with modified region was chemically etched in a $5 \% \mathrm{HF}$ acid solution. An ultrasonic bath was used to enhance the penetration of HF acid and the removal of debris. The etching process can be monitored by examining the transmission or reflection spectrum of the FBG. A total loss $7 \mathrm{~dB}$ was noticed after the etching process was finished. Thank to the tiny focal volume (around $1 \mu \mathrm{m}^{3}$ ) of the laser, a very thin layer of the glass was modified and with the subsequent etching, a micro-slot through the fiber with the opening of $1.2 \mu \mathrm{m}$ height was fabricated as Fig. 1(c) shows, revealing a high contrast ratio of 100:1.

\section{Fabrication and characterization of HWG}

Though a range of polymers can be used for filling, we used the UV-curable epoxy for fiber coating (Desolite 950200 from DSM Desotech Inc) for the initial work and cured it using fiber coating machine. Figure 2(a) gives the reflection spectrum of the device at different stage of the experiment. Before infusion, there was only one main Bragg peak at $1548 \mathrm{~nm}$, while after filling, a new peak appeared at longer wavelength around $1583 \mathrm{~nm}$. It further moved to $1599 \mathrm{~nm}$ when the epoxy was cured by UV light. As explained in [7], with high refractive index material filled in the micro-slot, a hybrid slab-waveguide with a polymer core and a glass cladding was formed which has higher effective refractive index than the optical fiber. Its evanescent wave extended to the neighbouring glass material, where the periodic grating structure still existed, giving rise to Bragg peaks of the slab waveguide. The further moving of the peak during UV curing process suggests increment of the refractive index when the epoxy turned into solid state. However, due to the large difference between the core and cladding after the epoxy was cured, light was so well confined in the polymer core region that the evanescent wave in the glass became so small that the influence of the grating was much reduced, making the Bragg reflection weakened.

The thermal response of the HWG in fiber was then investigated. Figure 2(b) shows its spectra for successively increased temperatures. As can be seen clearly, the Bragg peak moves to shorter wavelength with increasing temperature, indicating decreasing of the refractive index of the epoxy. In the mean time, the peak becomes stronger, caused by more portion of the light in the glass cladding. Figure 2(c) gives the variation of the Bragg wavelength 
with respect to the temperature, revealing an almost linear relation with a coefficient of $211 \mathrm{pm} /{ }^{\circ} \mathrm{C}$. The strain response was also tested and the result is shown in Fig. 2(d), giving an almost linear relation with a coefficient about $1.3 \mathrm{pm} / \mu \varepsilon$, similar to that of normal FBGs.

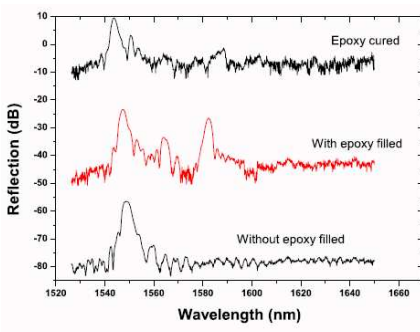

(a)

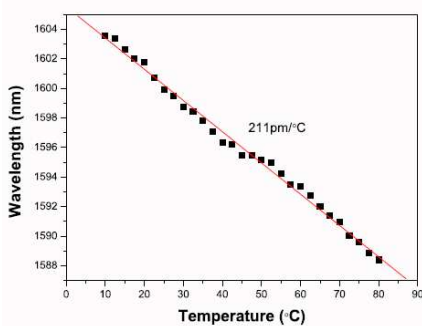

(c)

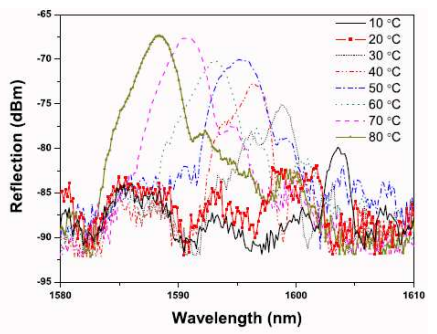

(b)

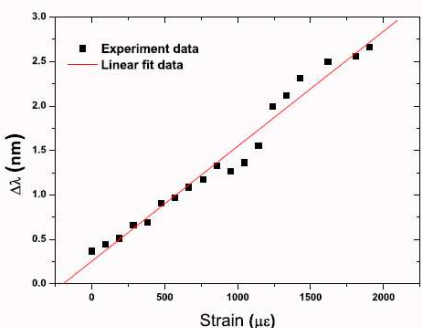

(d)

Fig. 2 (a) Reflection spectrum of the Bragg grating at different stages during UV curing of the epoxy. (b)Evolution of the spectrum of the hybrid waveguide grating when the temperature varied. (c) Relationship between the Bragg Wavelength and the temperature. (d) Relationship between the Bragg Wavelength and the strain.

\section{Discussion and conclusion}

Typical FBGs at $1550 \mathrm{~nm}$ in SMF-28 have thermal coefficient of $10 \mathrm{pm} /{ }^{\circ} \mathrm{C}$ and strain coefficient around $1 \mathrm{pm} / \mu \varepsilon[8]$. The results shown in fig.2(c) and (d) illustrate that the HWG presents more than 20x bigger thermal sensitivity than common FBGs in glass fiber while its sensitivity to strain is similar to that of FBG in glass fiber. By assuming factors of $M$ have similar values in equation (1) and then enlarging $K_{T, 2}$ by 20 times in equation (2), we can find that using such an HWG/FBG sensor, the resolution may be improved by $20 \times$ for the temperature and $2 \times$ for the strain.

In conclusion, we propose to use a HWG in conjunction with FBG strain sensor for improvement of resolution. The HWG was fabricated by infusion and followed curing of epoxy into a $1.2 \times 500 \mu \mathrm{m}$ micro-slot across an FBG which was made by fs laser patterning and chemical etching. The HWG was characterized in terms of strain and thermal responses, giving linear coefficient of $211 \mathrm{pm} /{ }^{\circ} \mathrm{C}$ and $1.3 \mathrm{pm} / \mu \varepsilon$ respectively. Analysis demonstrates that the resolution of the sensor is improved $20 \times$ for temperature and $2 \times$ for strain.

\section{Reference:}

[1] M.G. Xu, J.-L. Archambault, L. Reekie and J.P. Dakin, Discrimination between strain and temperature effects using dual-wavelength fibre grating sensors, Electron. Lett. 30 (1994) (13), pp. 1085-1087

[2] P.M. Cavaleiro, F.M. Araújo, L.A. Ferreira, J.L. Santos and F. Farahi, Simultaneous measurement of strain and temperature using Bragg gratings written in germanosilicate and boron-Codoped germanosilicate fibres, IEEE Photon. Technol. Lett. 11 (1999) (12), pp. $1635-1637$.

[3] Frazao, R. Romero, F.M. Araujo, L.A. Ferreira, J.L. Santos, Strain-temperature discrimination using a step spectrum profile fibre Bragg grating arrangement, Sensors and Actuators A: PhysicalVolume 120, Issue 2, , 17 May 2005, Pages 490-493.

[4] H.J. Patrick; G.M. Williams; A.D. Kersey; J.R. Pedrazzani; A.M. Vengsarkar, "Hybrid fiber Bragg grating/long period fiber grating sensor for strain/temperature discrimination," Photonics Technology Letters, IEEE, vol.8, no.9, pp.1223-1225, Sept. 1996

[5] Z. Xiong, G. Peng, B. Wu, and P. Chu, "Highly tunable bragg gratings in single-mode polymer optical fibers," Photonics Technology Letters, IEEE 11, 352-354 (Mar 1999)

[6] Y. Lai, K. Zhou, K. Sugden, and I. Bennion, "Point-by-point inscription of first-order fiber Bragg grating for C-band applications," Opt. Express 15, 18318-18325 (2007)

[7] K. Zhou, Y. Lai, X. Chen, K. Sugden, L. Zhang, and I. Bennion, "A refractometer based on a micro-slot in a fiber bragg grating formed by chemically assisted femtosecond laser processing," Opt. Express 15, 15848-15853 (2007).

[8] Hill, K.O.; Meltz, G., "Fiber Bragg grating technology fundamentals and overview," Lightwave Technology, Journal of , vol.15, no.8, pp.1263-1276, Aug 1997 